\title{
THE AIRSAR/TOPSAR INTEGRATED MULTI-FREQUENCY POLARIMETRIC AND INTERFEROMETRIC SAR PROCESSOR
}

\author{
Jakob J. Van Zyl, Anhua Chu, Scott Hensley, Yunling Lou, Yunjin Kim, Soren N. Madsen \\ Jet Propulsion Laboratory, California Institute of Technology, 4800 Oak Grove Drive \\ Pasadena, California 91109 \\ emailjacobv@blacks.jpl.nasa.gov, fax: (818) 393-5285,phone: (818) 354-1365
}

\section{INTRODUCTION}

Research on the utility at Synthetic Aperture Radar (SAR) data in many diverse applications has seen great activity in the past decade. In particular, two powerful SAR techniques emerged during this time. Polarimetric SARs were first demonstrated during the 1980's and is described by van Zyl et al. [1] and Zebker et al. [2]. Since then polarimetric SAR data have been applied to most disciplines of Earth Science. A recent summary of these investigations can be found in [3]. The second powerful SAR technique that has received much attention lately is SAR interferometry. While first published by Graham [4] in the 1970's, interest in this technique has steadily increased since the demonstration of SAR interferometry using digital processing [5]. For a summary of the different applications of SAR interferometry, please see [6].

To really derive the maximum information content from SAR data, it is desirable to combine polarimetric and interferometric techniques, preferably at multiple frequencies. The NASA/JPL AIRSAR/TOPSAR system [14] is capable of simultaneously acquiring interferometric (C- and/or L-band) and polarimetric data (C-, L- and Pband). In this paper we describe a processing approach that is used to process data acquired in these modes. This approach is different from that described by Madsen et al. [7] in both the motion compensation approach, as well as in the sense that data are deskewed before interferograms are formed. Using this approach, the same basic SAR processor is used to process data acquired in any of the modes supported by the AIRSAR/TOPSAR system.

\section{THE AIRSAR/TOPSAR SYSTEM}

The NASA/JPL AIRSAR system is a three-frequency airborne SAR system that was developed to be a general test-bed for advanced SAR techniques. The SAR system is flown on a NASA DC-8 passenger jet operated by NASA's Ames Research Center in Mountainview, California. The AIRSAR antennas are not gimaballed; instead the dual-polarized microstrip antennas are mounted fixed to the body of the DC-8 aft of the left wing. The earliest mode implemented in the AIRSAR system (operational since 1988) was the three-frequency polarimetric mode, where fully polarimetric data are acquired simultaneously at C-band, L-band and P-band. This mode was used to provide prototype data for the SIR-C/X-SAR science team and many of the algorithms applied to SIR-C data were developed using AIRSAR data.

In 1990 NASA, in collaboration with an Italian consortium (CORISTA), approved the addition of another set of C-band antennas to implement a single-pass, fixed baseline cross-track interferometer (XTI) for topographic 
mapping. The C-band antennas were provided by CORISTA, while NASA sponsored the system modifications and processor development described by Madsen et al. [7]. This mode of the AIRSAR system became known as TOPSAR [8] and data have been acquired since 1991. The original TOPSAR processing software has been updated several times since the original publication in [7]. One version of this updated software was delivered to the Environmental Research Institute of Michigan (ERIM) under a contract with the Defence Advance Research Projects Agency (DARPA), and is currently being used to process the data from the ERIM IFSARE system. In 1995 TOPSAR was extended to acquire XTI data simultaneously at C-band and L-band [9]. All TOPSAR interferometers can be operated in single or dual baseline modes. For single baseline operation signals are transmitted out of one antenna only, and the received signals are measured simultaneously through two antennas. In the dual baseline mode, signals are alternately transmitted out of the antennas at either end of the baseline, while the received signals are measured simultaneously through both antennas.

\section{PROCESSING APPROACH}

The aim of the Integrated AIRSAR Processor is to implement a processing strategy capable of processing all the modes described above with the same basic processor. Therefore, the processor must automatically produce coregistered multi-frequency images, whether or not at least one frequency was acquired in the interferometric mode. Madsen et al. [7] describe a way to process single frequency cross-track interferometry data. In this approach, the individual images are never explicitly deskewed. Rather, the deskew forms an integral part of the location algorithm; the along-track offset given by Madsen et al. [7] is identical to the deskew used in the traditional range-Doppler processor [10].

This follows from the fact that in traditional (non-interferometric) SAR processing, it is assumed that the imaged pixel is located at the intersection of the Doppler cone (centered on the velocity vector), the range sphere (centered at the antenna) and an assumed reference plane. Since the Doppler cone has its apex at the center of the range sphere, and its axis of symmetry is aligned with the velocity vector, it follows that all points on the intersection of the Doppler cone and the range sphere lie in a plane orthogonal to the velocity vector. The additional information provided by the interferometry is that the imaged point also has to lie on the cone described by a constant phase, which means that one no longer has to assume an arbitrary reference plane. This cone of equal phase has its axis of symmetry aligned with the interferometer baseline and also has its apex at the center of the range sphere. It then follows that the imaged point lies at the intersection of the Doppler cone, the range sphere and the equal phase cone. Using the same argument as before, it is clear that the point still lies in a plane orthogonal to the velocity vector. This along-track offset can still be calculated using the traditional expression for the deskew, as shown in Madsen et al. [7].

When processing multi-frequency data, one has two options to ensure that the output images automatically coregister. In the first approach, the sub-patch size is chosen such that the same number of output lines are kept for all frequencies. (This assumes that the radars operate at the same pulse repetition frequency, which is the case for the AIRSAR system.) All frequencies can then be processed with properly scaled Doppler parameters, meaning that the along-track shift for all frequencies will be the same. Therefore, the location parameters derived at any frequency can directly be applied to all the other frequencies. In this case, the size of the subpatch used, as well as the amount of overlap between adjacent sub-patches are determined by the length of the azimuth reference functions at the lowest frequency.

There are two main disadvantages to this approach. First, the fixed number of output lines for all frequencies means that the processor becomes quite inefficient in the case of the high frequencies for which the azimuth reference functions are much shorter than at the low frequencies, since large parts of the overlap areas between 
successive sub-patches are unnecessarily recalculated. Secondly, since the size of the patch at the high frequencies is large compared to the synthetic aperture size, the motion compensation may be less than optimum.

These disadvantages can be overcome by processing each frequency separately into the zero squint geometry before utilizing any interferometric information. After the deskew is applied, the multi-frequency images automatically co-register. This now means that each frequency can be processed with a sub-patch size optimally chosen for that particular frequency. In a sense this follows the same processing paradigm usually employed in repeat-pass interferometry. The disadvantage, at least in our implementation, is that the bookkeeping of the phases during motion compensation is slightly more complicated.

The post processing steps applied after the SAR processing depends on the mode in which the data were acquired. For the three-frequency polarimetric mode the various cross-products are combined into the standard AIRSAR compressed Stokes Matrix format and then radiometrically calibrated.

In the case where interferometric data were acquired, the interferogram phase is first unwrapped and the slant range digital elevation model is formed. The location parameters for each slant range pixel is now calculated using the elevation model just derived. Since all the images acquired at the different frequencies now are coregistered, the same set of locations parameters an be used to geometrically resample all images. These location parameters are then used to resample the calibrated polarimetric SAR images. In addition to the calibrated and geometrically corrected SAR images and the digital elevation model, images of the local incidence angle and the interferometric correlation coeffiecient are also provided. These images are also geometrically corrected using the location parameters calculated from the interferometric phase information.

\section{RESULTS}

The performance of the TOPSAR instrument and processor was previously reported by Madsen, et al. [7], who compared the radar derived digital elevation models with ones derived using conventional optical stereo techniques. Their analysis showed the difference between the DEMs to be $2.2 \mathrm{~m} \mathrm{r} . \mathrm{m} . \mathrm{s}$ in relatively flat terrain, and up to $5.0 \mathrm{~m}$ in mountainous areas. Instead of repeating these analyses here, we compare the results of the Integrated Processor described here to that of the TOPSAR processor described by Madsen et al. [7].

To compare the results of the processors, we processed two strips of data through both processors, and then compared the results. First we tested the relative geometrical accuracy of the two resulting data sets. This is done by cross-correlating small subsections of the images, and measuring the along-track and cross-track offsets between the images. This analysis is repeated for a grid of 100 points spaced uniformly through the images. To test the effect of relief on the results, this comparison was done for a flat area covering the Bolivar peninsula near Galveston, Texas, and a mountainous area covering part of Mount Rainier in Washington State. The total relief in the Bolivar scene is less than $50 \mathrm{~m}$ while the total relief in the Mount Rainier scene is about $1500 \mathrm{~m}$. The results of the relative geometry test shows the rms difference between the Bolivar images to be $1.3 \mathrm{~m}$ in the cross-track direction, and $2.1 \mathrm{~m}$ in the along-track direction for a nominal post spacing of $10 \mathrm{~m}$. For the Mount Rainier scenes, the rms difference are $2.3 \mathrm{~m}$ and $4.9 \mathrm{~m}$ in the cross- and long-track directions respectively. This confirms previous reports that results for relatively flat areas are typically better than those for high relief areas. 


\section{REFERENCES}

[1] J. J. van Zyl., H.A. Zebker and C. Elachi, "Imaging radar polarization signatures: Theory and observations," Radio Science, 22, pp. 529--543, 1987.

[2] H. A. Zebker, J. J. van Zyl and D. N. Held, "Imaging radar polarimetry from wave synthesis," Journal of Geophysical Research, 92, no. B1, pp. 683--701, 1987.

[3] J. J. van Zyl, "SAR polarimetry and interferometry," in Review of Radio Science 1993-1996, R.W. Stone (Ed), Oxford University Press, New York, 1996.

[4] L. C. Graham, "Synthetic Interferometer Radar for Topographic Mapping,” Proc. IEEE, 62, pp.763-768, 1974.

[5] H. Zebker and R. Goldstein, “Topographic Mapping from Interferometric SAR Observations,” J. Goephys. Res., 91, pp. 4993-4999, 1986.

[6] C. T Allen, "Interferometric Synthetic Aperture Radar," IEEE Geoscience and Remote Sensing Society Newsletter, pp. 6-13, September 1995.

[7] S. N. Madsen, H. A. Zebker, and J. Martin, “Topographic Mapping Using Radar Intereferometry: Processing Techniques,” IEEE Trans. Geosci. Remote Sens., GRS-31, pp. 246-256, 1993.

[8] H. A. Zebker, S. N. Madsen, J. Martin, K. B. Wheeler, T. Miller, Y. Lou, G. Alberti, S. Vetrella, and A. Cucci, “The TOPSAR interferometric Radar Topographic Mapping Instrument,” IEEE Trans. Geosci. Remote Sens., GRS-30, pp. 933940, 1992.

[9] J.J van Zyl., H.A. Zebker, S. Hensley and D.R. Haub, “The new dual frequency (C- and L-band) TOPSAR airborne interferometric SAR,” Proceedings of IGARSS'95, Florence, Italy, July 10-14, 1995.

[10] J. C. Curlander and R. N. McDonough, Synthetic Aperture Radar Sytems and Signal Processing, John Wiley and Sons, New York, 1991. 